\title{
HIPERGLUCEMIA DE ESTRÉS EN INFARTO AGUDO DE MIOCARDIO: ¿SE ASOCIA CON AUMENTO DE MORTALIDAD? TAMBIÉN UNA VALORACIÓN DE LA CONDUCTA TERAPÉUTICA
}

\author{
STRESS HYPERGLYCEMIA IN ACUTE MIOCARDIAL INFARCTION: \\ IS IT ASSOCIATED WITH INCREASED MORTALITY? \\ A CONCERN OF THERAPEUTIC BEHAVIOR TOO
}

\begin{abstract}
Guillermo Alzueta', Juan Carlos Bauchi', Guillermo Dieuzeide', Darío Rímoli', Raúl Suárez', Amílcar Sosa ${ }^{1}$, Carlos Santillán (h) ${ }^{1}$, Mirta Linares ${ }^{1}$, Ricardo Denaroํ, Gracieta Zarza ${ }^{1}$, Adrián Decundo ${ }^{1}$, Olguita Vera ${ }^{1}$, Rosa Salazar', Segundo Guzmán Rodríguez ${ }^{1}$, Abel Weinmeister ${ }^{1}$, Rodolfo Dettorre' ${ }^{1}$ Karina Bastone', Alfredo Caccavo' ${ }^{1}$, Horacio Costa ${ }^{1}$, Claudio González ${ }^{1,2}$
\end{abstract}

\section{RESUMEN}

Introducción: la hiperglucemia de estrés puede aumentar el riesgo de muerte en infarto agudo de miocardio (IAM).

Objetivos: en una muestra se observó retrospectivamente la relación entre la hiperglucemia y la mortalidad en IAM, y también se consideró qué conducta terapéutica se adoptaba en los pacientes hiperglucémicos.

Materiales y métodos: se evaluó la mortalidad al alta en 349 pacientes que ingresaron por IAM en 11 hospitales entre mayo de 2000 y abril de 2001.

Resultados: $n=349$ pacientes. Edad: $64,3 \pm 12,9$ años. Glucemia al ingreso: $158,8 \pm 96 \mathrm{mg} / \mathrm{dl}$. Score de Killip =ó> 2 en el $25 \%$. La mortalidad global fue del 19,1\%. En el análisis univariado se asoció con edad ( $p<0,001)$, score de Killip $(p<0,001)$, arritmias en la internación $(p<0,001)$ y glucemia $(p<0,001)$. Se halló relación inversa con la tensión arterial (TA) $(p<0,001)$. En el análisis multivariado la mortalidad al egreso se asoció a: edad (OR: 1,03 por año IC95\% 1,00-1,06, $p=0,03$ ), score de Killip (OR: 3,93 por punto, IC95\% 2,63-5,87, $p<0,001$ ), presencia de arritmias (OR=3,49 IC95\%: 1,64-7,43, $p=0,001)$, glucemia al ingreso $>177 \mathrm{mg} / \mathrm{dl}$ (Qs) (OR=2,87, IC95\% 1,34-6,17, $\mathrm{p}=0,007)$. De la muestra $79,6 \%$ era no diabético. La diferencia de mortalidad en no diabéticos hiperglucémicos y no hiperglucémicos fue significativa $(40,5 \%$ vs $14,1 \% p<0,001)$. El efecto de la hiperglucemia fue significativa en pacientes con score de Killip 2 (10,8\% en los no hiperglucémicos vs $55 \%$ en los hiperglucémicos $[p<0,05])$. Considerando el tratamiento de la glucemia en internación, la media de glucemia del grupo tratado fue de $265,6 \pm 120,4 \mathrm{mg} / \mathrm{dl}$ mientras que la del grupo sin tratamiento fue $131,6 \pm 61,6 \mathrm{mg} / \mathrm{dl}(\mathrm{p}<0,001)$. En los tratados la glucemia media fue un $50 \%$ más alta que la media considerada para hiperglucemia. Sólo el $64,4 \%$ de los pacientes en el cuartilo superior (177 mg/dl) recibió tratamiento de su glucemia, el 89,6\% de ellos insulina (el 20,3\% por vía endovenosa) y un $3,6 \%$ de los pacientes hiperglucémicos (Qs) tratamiento con drogas orales. Los pacientes que recibieron insulina sub-

\section{ABSTRACT}

Introduction: stress hyperglycemia could increase mortality after acute myocardial infarction (AMI).

Objectives: we explored the association between hyperglycemia and mortality after AMI in a retrospective cohort study. We consider the treatment behavior of hyperglycemia too.

Materials and methods: the mortality rate at hospital discharge post AMI was obtained from a sample of 349 patients assisted in 11 hospitals, between May 2000 and April 2001.

Results: $N=349$. Age: $64.3 \pm 12.9$. Glycemia at admission: $158.8 \pm 96.0 \mathrm{mg} / \mathrm{dl}$. Killip score $>2$ in $25 \%$ of the subjects. Global mortality rate was $19.4 \%$. In the univariate analysis, mortality was associated to age $(p<0.001)$, Killip score $(p<0.001)$, arrhythmia $(p<0.001)$ and glycaemia at admission $(p<0.001)$. Mortality was inversely correlated to BP $(p<0.001)$. In the multivariate analysis, mortality was associated to age $(O R=1.03$ per year; $95 \% \mathrm{Cl}=1.00-1.06, p=0.03)$, Killip score $(O R=3.93$ per point; $95 \% \mathrm{Cl}=2.63-5.87, p<0.001)$, arrhythmia (OR=3.49; 95\% Cl=1.64-7.43, $p=0.001)$ and glycaemia at admission equal or higher than the upper quartile [177 $\mathrm{mg} / \mathrm{dl}]$ (OR=2.87; $95 \% \mathrm{Cl}=1.34-6.17, \mathrm{p}=0.007)$. From the total sample $79.6 \%$ were no diabetics. In these patients mortality was statistically significant in hyperglycemic versus non-hyperglycemic $140.5 \%$ vs $14.1 \%, p<0.001)$. The effect of hyperglycemia was significant in patients admitted in Killip 2 (moderate heart failure) $110.8 \%$ in non-hyperglycemic vs $55 \%$ in hyperglycemic patients. [ $p<0.005])$. If we consider the treatment of glycaemia, the mean value of glycaemia in the treated group was $265.6 \pm 120.4 \mathrm{mg} / \mathrm{dl}$ and in untreated group it was 131.6 \pm 61.6 $\mathrm{mg} / \mathrm{dl}(\mathrm{p}<0.001)$. That mean value of glycaemia in treated patients was 50\% higher than the value of glycaemia that we consider as hyperglycemic. Only $64.4 \%$ of the patients with glycaemia at upper quartile $(177 \mathrm{mg} / \mathrm{dl})$ received treatment to control glycaemia, $89.6 \%$ of them were treated with insulin (20.3\% intravenously) and $3.6 \%$ of patients with hyperglycemia received oral pharmacological agents to treat it. The mean 
cutánea tenían una glucemia media de 155,6 1110,9 mg/dl, mientras que quienes la recibieron por venoclisis su glucemia media fue de $278,5 \pm 120,3 \mathrm{mg} / \mathrm{dl}(\mathrm{p}<0,0001)$. Se trataron 41 de $50(82 \%)$ pacientes que se conocían diabéticos y tenían hiperglucemia, mientras que fueron tratados sólo 10 de 27 pacientes $(41,8 \%)$ sin diabetes conocida a pesar que también estaban hiperglucémicos.

Conclusiones: la mortalidad se asoció positivamente con el sexo, la edad, score de Killip, arritmia en internación e hiperglucemia al ingreso $>\mathrm{Qs}$, y en forma inversa con TA. Dentro de los pacientes con hiperglucemia la mortalidad fue, comparativamente con los no hiperglucémicos, muy superior en los no diabéticos comparado con los diabéticos. El efecto de la hiperglucemia fue especialmente evidente en los pacientes admitidos con score de Killip 2.

Aproximadamente una tercera parte de los pacientes hiperglucémicos no recibió tratamiento para ello. El 90\% de los pacientes tratados recibió insulina y sólo un $20 \%$ por vía intravenosa. Los valores de glucemia tomados en cuenta para iniciar tratamiento fueron un $50 \%$ más elevados que el valor considerado en nuestro estudio como hiperglucemia. La conducta terapéutica se enfocó en intervenir la glucemia de los diabéticos conocidos más que en el valor de la misma ( $82 \%$ vs $41,8 \%$ ) aunque los pacientes estuvieran hiperglucémicos.

Palabras clave: hiperglucemia de estrés; mortalidad; infarto agudo de miocardio.

Revista de la Sociedad Argentina de Diabetes 2018; Vol. 52 (37-47) value of glycaemia in patients who received insulin by subcutaneous route was $155.6 \pm 110.9 \mathrm{mg} / \mathrm{dl}$ and the mean value of glycemia in those that received intravenous insulin treatment was $278.5 \pm 120.3 \mathrm{mg} / \mathrm{dl}(\mathrm{p}<0.0001)$. There was treated 41 of 50 patients (82\%) that with known diabetes that were hyperglycemic (>177 mg/dl), while was treated only 10 of 27 pacientes $(41.8 \%)$ with unknown diabetes although they were hyperglycemic too.

Conclusions: post-acute myocardial infarction mortality was significantly associated to age, Killip score, arrhythmia and glycaemia at admission above the upper quartile $(177 \mathrm{mg} / \mathrm{dl})$. It was inversely associated to blood pressure values. Mortality was higher in hyperglycemic patients with and without diabetes. The effect of hyperglycemia was especially noticed in patients admitted with moderate heart failure (Killip score 2). Approximately one third of patients remained untreated in spite of being clearly hyperglycemic. $90 \%$ of hyperglycemic patients received insulin and only 20\% received insulin intravenously. Glycemic values taken into account to prescribe insulin treatment were $50 \%$ higher than hyperglycemic value considered. The therapeutic behavior was to treat mainly diabetics patients in despite of non-diabetic patients $182 \%$ vs. $41.8 \%$ respectively) although they were both hyperglycemic.

Key words: stress hyperglycemia; mortality; acute myocardial infarction.

Revista de la Sociedad Argentina de Diabetes 2018; Vol. 52 (37-47)
1 Capítulo Atlántico de la Sociedad Argentina de Diabetes (ATLANSAD): Balcarce, Punta Alta, Chacabuco, Junín, Mar del Plata, Bahía Blanca, Olavarría, Coronel Suárez, Puerto Madryn, Las Flores, Río Gallegos

2 Departamento de Farmacología de la Universidad Nacional de Buenos Aires, Ciudad Autónoma de Buenos Aires, Argentina
Contacto del autor: Dr. Guillermo Alzueta

E-mail: g_alzueta@yahoo.com.ar

Correspondencia: Av. René Favaloro № 782, (CP7620)

Balcarce, Provincia de Buenos Aires, Argentina

Fecha de trabajo recibido: 31/07/18

Fecha de trabajo aceptado: 28/08/2018

Conflictos de interés: los autores declaran que no existe conflicto de interés 


\section{INTRODUCCIÓN}

Desde hace varios años se observa que un número elevado de pacientes con infarto agudo de miocardio (IAM) presenta hiperglucemia'. Desde esa primera descripción se realizaron investigaciones de glucemia elevada en pacientes con IAM que en algunos casos llegaban hasta el 50\% o más, varios de ellos sin diagnóstico de diabetes previamente. En el año 1987 se efectuó la observación de la asociación de mortalidad aumentada en pacientes con IAM que ingresaban con hiperglucemia ${ }^{2}$. Los mecanismos de tal asociación son poco claros aún, pero desde el hallazgo realizado en el estudio DIGAMI en $1995^{3}$, en el cual Malmberg et al. lograron reducir la mortalidad descendiendo la glucemia con tratamiento insulínico intensificado, se infirió que la asociación entre hiperglucemia de estrés y el incremento de mortalidad por IAM no es casual.

En el año 2000 se publicó un metaanálisis que analizó los datos de 15 estudios en los que se halló que la hiperglucemia de estrés al inicio del IAM se asociaba a un riesgo aumentado de muerte y falla de bomba, tanto en diabéticos como en no diabéticos, siendo en estos últimos llamativamente mayor la tasa de mortalidad 4 .

Fue entonces que nos planteamos observar, con un estudio retrospectivo, la relación existente entre la hiperglucemia de estrés y la mortalidad en IAM en una muestra de pacientes de nuestro país.

\section{MATERIALES Y MÉTODOS}

En un análisis retrospectivo se evaluó la mortalidad al alta hospitalaria de 349 pacientes, de entre 25 y 79 años, con diagnóstico de IAM, ingresados en 11 hospitales generales de las localidades de Balcarce, Punta Alta, Chacabuco, Junín, Mar del Plata, Bahía Blanca, Olavarría, Coronel Suárez, Puerto Madryn, Las Flores y Río Gallegos, ubicadas dentro del territorio del Capítulo Atlántico de la Sociedad Argentina de Diabetes, desde el $1^{\circ}$ de mayo de 2000 al 30 de abril de 2001.

\section{Pacientes}

Se evaluaron las historias clínicas de 349 pacientes ingresados en dichos hospitales, dentro de las fechas indicadas, con diagnóstico de infarto agudo de miocardio (IAM) según los criterios detallados más adelante. De la historia clínica se valoraron: al ingreso medición de tensión arterial sistólica (TAS) y tensión arterial diastólica (TAD), glucemia, arritmias, y score de Killip y Kimbal registrados en la historia clínica al ingreso o en las primeras 6 hs del ingreso.

\section{Infarto agudo de miocardio}

Se consideró infarto agudo de miocardio (IAM) cuando se diagnosticó por dos de los siguientes tres criterios: a) clínico: dolor precordial típico o atípico de más de 30 minutos de duración; b) electrocardiográfico: cambios evolutivos del segmento ST y onda T (STT) con aparición de nuevas ondas $\mathrm{Q}$; si no se halló onda Q (IAM no Q) el diagnóstico se efectuó por los otros dos criterios; c) enzimático: curva enzimática típica de CPK, TGO y LDH.

\section{Historia clínica}

Se consideraron antecedentes de diabetes mellitus (DM), hipertensión arterial (HTA), dislipidemia y hábito tabáquico. Se registró la glucemia (siempre que esté fuera de una muestra del ingreso o no más allá de las 6 hs del mismo), TAS y $T A D$, caras del corazón afectadas por el IAM, presencia de arritmias tanto al ingreso como después de las primeras 24 hs, y score de Killip y Kimbal valorado en las primeras 6 hs del ingreso.

- Arritmias: se valoró dentro de ellas fibrilación ventricular (FV), taquicardia ventricular (TV) y bloqueos aurículoventriculares de $2^{\circ}$ grado $\left(\mathrm{BAV}^{\circ}\right.$ ) y $3^{\circ}$ grado $\left(\mathrm{BAV}^{\circ}\right)$.

- Score de Killip y Kimbal: se consideró según escala de Killip Kimbal: a) clase 1: sin ningún signo de congestión pulmonar o venosa; b) clase 2: con insuficiencia cardíaca moderada evidenciada por rales crepitantes en ambas bases pulmonares, galope de tercer ruido, taquipnea o signos de insuficiencia cardíaca derecha con congestión hepática y venosa; c) clase 3: con insuficiencia cardíaca grave y edema pulmonar; d) clase 4: con shock y presión sistólica menor de $90 \mathrm{mmHg}$ y signos de vasoconstricción periférica, cianosis periférica, confusión mental y oliguria.

- Glucemia: se tuvo en cuenta el primer valor de glucemia que constara en la historia clínica siempre que se obtuviera dentro de las primeras 6 hs del ingreso y no hubiera mediado un tratamiento que pudiera alterarla.

- Tratamiento de la glucemia: también se consideró la conducta del tratamiento que se tomaba ante los valores de glucemia hallados. Se evaluaron las drogas y las vías utilizadas para ese tratamiento, 
así como las medias de los valores y los grupos en que se tomaba tal o cual conducta terapéutica.

\section{Análisis estadístico}

La naturaleza de la distribución de las variables cuantitativas se exploró a través del test de Shapiro-Wilk. Las diferencias entre grupos de datos cuantitativos se evaluaron por medio de los tests: t de Student, $U$ de Mann-Whitney, ANOVA de una vía o Kruskal-Wallis según la distribución. Las diferencias entre grupos de datos cualitativos se estudiaron a través de $\mathrm{Chi}^{2} \mathrm{o}$ del test de Fisher. Las asociaciones univariadas se determinaron a través de la correlación de Spearman o del empleo de modelos de regresión logística univariada. La regresión logística múltiple (máxima verosimilitud, quasi Newton) se empleó para el análisis multivariado. Se consideraron significativos los valores de $p<0,05$. Software: CSS/Statistica, 1993.

\section{RESULTADOS}

En este estudio retrospectivo se evaluó la mortalidad al alta hospitalaria de 349 pacientes, con edades de entre 25 y 79 años, que ingresaron con diagnóstico de IAM en 11 hospitales generales de las ciudades argentinas ubicadas en el territorio del Capítulo Atlántico de la Sociedad Argentina de Diabetes. La media de edad fue de $64,3 \pm 12,9$ años. El 78,5\% era varón y el $21,8 \%$ mujer. La media de glucemia fue de $158,8 \pm 96 \mathrm{mg} / \mathrm{dl}$. El cuartilo superior de glucemia (Qs) fue de $177 \mathrm{mg} / \mathrm{dl}$. El $25 \%$ de los pacientes presentó un valor de score de Killip igual o superior a 2. La media de tensión arterial sistólica (TAS) fue de 132,5 $\pm 44,8 \mathrm{mmHg}$ y la media de tensión arterial diastólica (TAD) de $79 \pm 19,1 \mathrm{mmHg}$. La media de la mortalidad global fue de $19,4 \%$.

En el análisis univariado la mortalidad al egreso de internación por IAM se asoció positivamente con: sexo: mayor en mujeres $(p=0,02)$. Edad: $r S=0,26 \quad(p<0,001)$. Hábito tabáquico: $r S=0,11$ $(p=0,04)$. Score de Killip: $r S=0,56(p<0,001)$. Arritmias en internación: $r S=0,36(p<0,001)$. Glucemia de ingreso: $r S=0,31(p<0,001)$. Se halló correlación inversa con TAS y TAD $(p<0,001)$. En el análisis multivariado la mortalidad al egreso de pacientes con IAM se asoció positivamente con: edad: OR: 1,03 por año de vida (IC95\%=1,00-1,06) $p=0,003$. Score de Killip: OR=3,93 por punto de score de Killip (IC95\% $=2,63-5,87) p<0,001$. La presencia de arritmias: OR=3,49 (IC95\%=1,64-7,43) $\mathrm{p}=0,001$.
Glucemia $\geq 177 \mathrm{mg} / \mathrm{dl}$ (Os): OR=2,87 (IC95\% = 1,34-6,17) $p=0,007$.

Se analizó la muestra según los pacientes tuvieran diagnóstico previo de diabetes o aquellos que no tuvieran diagnóstico de diabetes al ingreso en el hospital. Los pacientes con diagnóstico previo de diabetes constituyeron el $20,4 \%$ de la muestra, mientras el $79,6 \%$ no tenía diagnóstico de diabetes al ingreso. De los diabéticos conocidos ingresó con glucemia $\geq 177 \mathrm{mg} / \mathrm{dl}$ (Os) el $71,5 \%$, y de los pacientes sin diagnóstico previo de diabetes a la internación ingresó con glucemia $\geq 177 \mathrm{mg} / \mathrm{dl}$ el 13,4\%. En los pacientes diabéticos conocidos la mortalidad general fue de $27,1 \%$, y entre ellos los que ingresaron con glucemia $\geq 177 \mathrm{mg} / \mathrm{dl}$ (Qs) tuvieron una mortalidad del 34\%, y en aquellos que ingresaron con glucemias $<177 \mathrm{mg} / \mathrm{dl}$ manifestaron una mortalidad del 10\% $(p=0,08)$. Por su parte en los pacientes sin diabetes conocida la mortalidad general fue del $17,6 \%$, y en ese grupo la mortalidad de los pacientes con glucemia de ingreso $\geq 177 \mathrm{mg} / \mathrm{dl}$ (Qs) fue del $40,5 \%$ y en los que ingresaron con glucemia inferior al cuartil superior (Qs) del 14,1\% ( $p<0,001)$ (Figura 1).

También se comparó la mortalidad de los pacientes con glucemia $\geq 177 \mathrm{mg} / \mathrm{dl}$ (hiperglucémicos) y los que tenían glucemia de ingreso $<177 \mathrm{mg} /$ dl (no hiperglucémicos) por cada punto de score de Killip. En el resultado de este análisis (Figura 2) se halló que para el punto 1 del score de Killip (sin insuficiencia cardíaca) la mortalidad general fue de $5,2 \%$, mientras que la mortalidad en no hiperglucémicos de 5,3\% y 4,5\% en hiperglucémicos. Para el punto 2 del score de Killip (insuficiencia cardíaca moderada) la mortalidad total fue de $26,3 \%$, mientras que en no hiperglucémicos fue de $10,8 \%$ y de $55 \%$ en los hiperglucémicos $(p<0,01)$. En el punto 3 del score de Killip (insuficiencia cardíaca severa) la mortalidad total fue de $62,8 \%$, mientras que en los no hiperglucémicos fue de $59,1 \%$ y en los hiperglucémicos de 69,2 $\%$. En el punto 4 del score de Killip (shock cardiogénico) la mortalidad global fue de $85,7 \%$, mientras que en los no hiperglucémicos fue de $75 \%$ y en los hiperglucémicos de $100 \%$.

En cuanto al tratamiento de la glucemia los hallazgos fueron los siguientes:

- De los 71 pacientes con diabetes conocida, 50 fueron hiperglucémicos (>177 mg/dl) y de este grupo $41(82 \%)$ recibieron tratamiento para su glucemia. Mientras tanto de los 278 pacientes no 
conocidos como diabéticos al ingreso, 27 ingresaron con hiperglucemia (>177 mg/dl) y de ellos solamente a $10(41,8 \%)$ se les trató su glucemia.

- La media de glucemia del grupo de pacientes no tratados (fueran diabéticos conocidos o no) fue de $131,6 \mathrm{mg} / \mathrm{dl} \mathrm{DS} \pm 61,6 \mathrm{mg} / \mathrm{dl}$ y la media de glucemia de los pacientes tratados fue $265,6 \mathrm{mg} / \mathrm{dl}$ $D S \pm 120,4 \mathrm{mg} / \mathrm{dl}(p<0,001)$. Este valor fue un $50 \%$ más alto que el considerado en nuestro estudio como hiperglucemia (>177 mg/dl).

- Solamente el $64,4 \%$ de los pacientes con glucemia en el cuartil superior (> $177 \mathrm{mg} / \mathrm{dl}$ ) recibió tratamiento de la misma. El 89,6\% de ellos recibió insulina y un 3,6\% tratamiento con drogas orales para su glucemia. Un $20,3 \%$ de los pacientes tratados con insulina recibió la misma por venoclisis, mientras que el resto por vía subcutánea.

- La media de glucemia de los que recibieron un plan insulínico por vía subcutánea fue de $264 \pm 121,7 \mathrm{mg} / \mathrm{dl}$ y aquellos que recibieron plan de insulina por venoclisis tenían una media de glucemia de $342,5 \pm 94,8 \mathrm{mg} / \mathrm{dl}(\mathrm{p}=0,002)$.

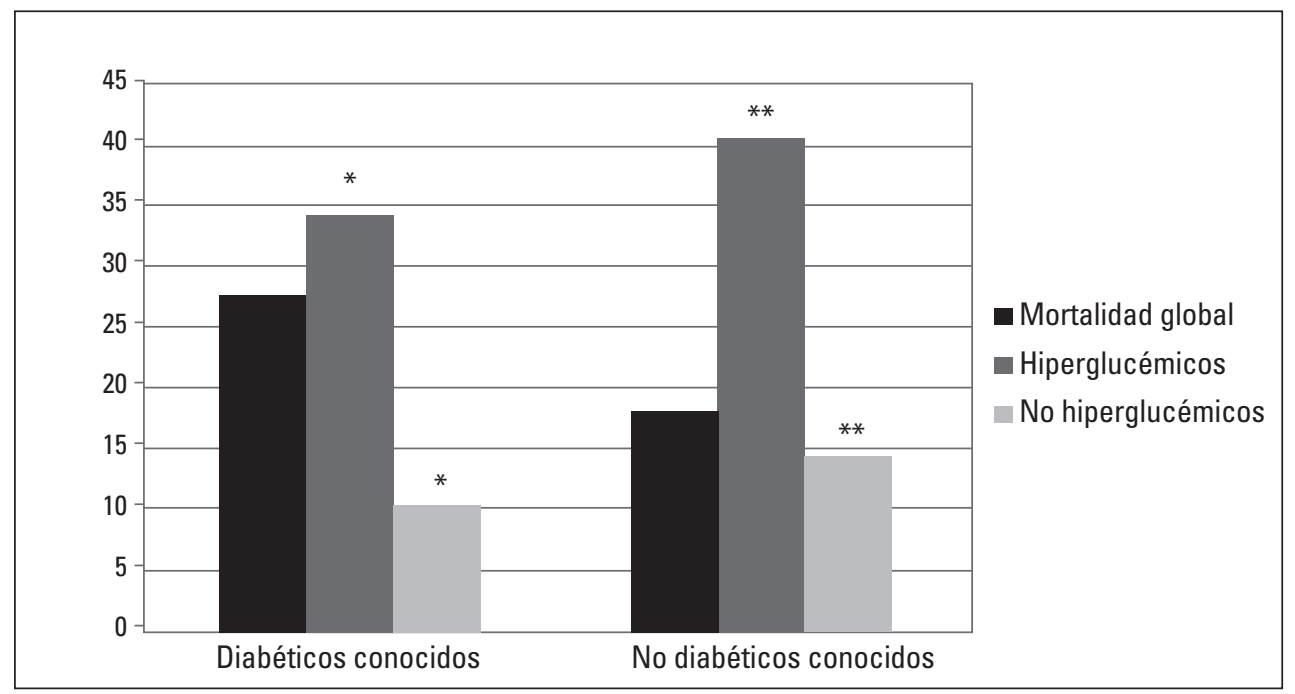

${ }^{*} p=0,08 ;{ }^{*} p<0,001$.

Figura 1: Evaluación de mortalidad total, en pacientes hiperglucémicos y no hiperglucémicos, valorando si eran diabéticos conocidos o no diabéticos conocidos al ingreso hospitalario.

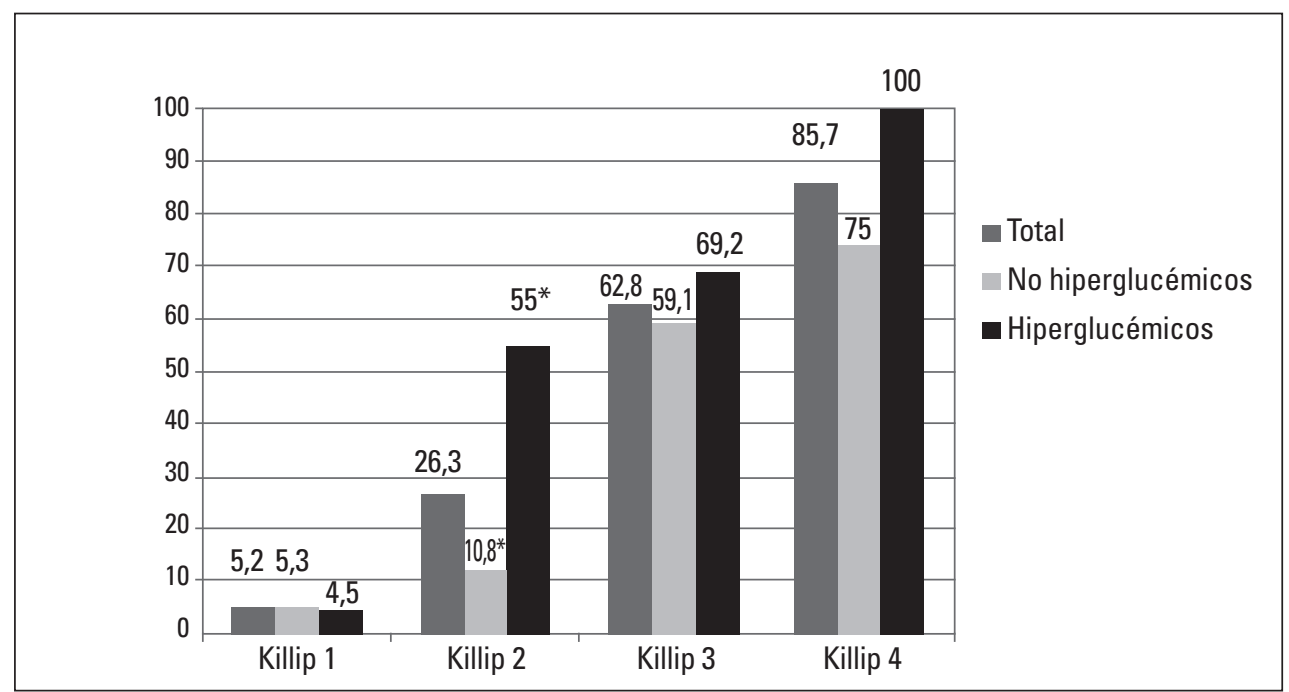

${ }^{*} p<0,01$.

Figura 2: Muestra la evaluación de mortalidad total, en pacientes no hiperglucémicos y en pacientes hiperglucémicos, por cada punto de la escala de Killip y Kimball. 


\section{DISCUSIÓN}

En nuestra muestra de 349 pacientes ingresados con diagnóstico de IAM, la mortalidad al alta hospitalaria se asoció positivamente a edad, score de Killip, la presencia de arritmias y glucemia de ingreso $\geq 177 \mathrm{mg} / \mathrm{dl}$ (cuartilo superior) y en forma inversa a la TAS y TAD. La mortalidad fue mayor en quienes ingresaron hiperglucémicos que en los no hiperglucémicos, tanto en pacientes con diagnóstico previo de diabetes como en los que no tenían diagnóstico de diabetes, pero sólo en estos últimos la diferencia fue estadísticamente significativa. Dada la característica retrospectiva de la muestra estudiada no pudo analizarse si entre ellos había pacientes que tenían diabetes no conocida o no registrada. Igualmente estos hallazgos coinciden con otros estudios que registraron características similares a las de nuestra muestra ${ }^{4}$.

Cuando se estudió la diferencia de mortalidad entre pacientes hiperglucémicos (glucemia de ingreso $\geq 177 \mathrm{mg} / \mathrm{dl}$ ) y no hiperglucémicos (glucemia $<177 \mathrm{mg} / \mathrm{dl}$ ) se encontró que salvo en el score de Killip 1 (ausencia de insuficiencia cardíaca), en el resto de las categorías la mortalidad fue mayor en los hiperglucémicos, siendo muy notoria (55\% vs $10,5 \%)$ y estadísticamente significativa en el punto 2 del score de Killip (insuficiencia cardíaca moderada) y mayor, pero no tan notoria, en los pacientes con score 3 de la escala de Killip $169,2 \%$ vs $59,1 \%$ ) y con score 4 de la escala de Killip $(100 \%$ vs $75 \%)$, mostrando ello una interesante observación dado que la diferencia fue muy notoria en pacientes con hiperglucemia en el grado de insuficiencia cardíaca menor donde no era esperable una tasa de mortalidad tan evidente como la hallada aquí (55\%). En los grados de insuficiencia cardíaca más severa (insuficiencia cardíaca severa [score 3 de Killip] y shock cardiogénico [score 4 de Killip]) tuvo más lógica hallar menos diferencia entre las categorías, ya que la sola presencia de una falla funcional cardiológica grave acarrea una mayor mortalidad per se, característica que le da más importancia al hallazgo realizado en los pacientes con score 2 de la escala de Killip.

Los resultados en esta muestra son similares a los hallados desde hace muchos años en diversos estudios, todos descriptos en el metaanálisis de Capes et al. ${ }^{4}$. Lo que no se encuentra descripto en esos resultados es la notoria diferencia de mortalidad entre pacientes hiperglucémicos y no hiperglucémicos en el score 2 de Killip que he- mos desarrollado anteriormente, mientras que se describe, en cuatro de los 15 trabajos del metaanálisis citado, un mayor riesgo de insuficiencia cardíaca y shock cardiogénico en los pacientes no diabéticos hiperglucémicos, hecho que hemos detectado en nuestra descripción por escala de score de Killip y Kimball.

Los mecanismos para explicar el mayor riesgo de mortalidad en pacientes hiperglucémicos vs no hiperglucémicos, independientemente de estar precedido de diagnóstico de diabetes, es aún una incógnita. Aún hoy no se conoce ciertamente si la hiperglucemia es un marcador de mayor agresividad de la enfermedad basal, debido a un aumento de las fuerzas contrarreguladoras que simplemente manifiestan el estrés de la patología como elevación de la glucemia o representan un factor patogénico que agrava la enfermedad basal ${ }^{5}$.

La glucemia ha demostrado acciones perjudiciales, sobre todo en estudios in vitro o en modelos animales en el sistema inmune ${ }^{6,7}$, en el funcionamiento del miocardio, con más evidencia en la isquemia ${ }^{8,9}$, en el sistema nervioso central ${ }^{10,11}$ y en la función endotelial ${ }^{12}$. Con respecto al tejido miocárdico en particular, en modelos experimentales se encontró una acción tóxica de los ácidos grasos libres en la zona isquémica lo que favorece la disfunción celular miocárdica y promueve el aumento de la demanda de oxígeno, hecho que reduce la contractilidad miocárdica con falla de bomba subsiguiente y aumenta la posibilidad de arritmias ${ }^{13,14}$.

La hiperglucemia de estrés se ha asociado a mortalidad en IAM, tanto en nuestra serie como en los trabajos anteriormente comentados ${ }^{4}$, y ese hecho se ha observado con más frecuencia (como también en nuestra muestra) en los pacientes sin diabetes al ingreso. Ello podría explicarse porque los diabéticos podrían estar más habituados a estar expuestos a los valores considerados como "hiperglucémicos" en esos trabajos y en el nuestro, lo que le conferiría una mayor tolerancia a las elevaciones de glucemia consideradas en esas muestras como hiperglucemia, sin que se produjeren los daños de la magnitud como los que sufrirían los pacientes sin diabetes. Este hecho ha sido remarcado por Mallmberg et al. en un subanálisis del estudio DIGAMI ${ }^{15}$ que mostró en los pacientes diabéticos una asociación más evidente con valores glucémicos extremadamente elevados (tercilo superior = glucemia $>325 \mathrm{mg} / \mathrm{dl}$ ), con una tasa de mortalidad que fue un $50 \%$ más alta que los del ter- 
cilo medio (glucemia entre $270 \mathrm{mg} / \mathrm{dl}$ y $325 \mathrm{mg} / \mathrm{dl}$ ) y tercilo bajo (glucemia < de $270 \mathrm{mg} / \mathrm{dll}$ ). Todos estos valores son mucho más altos que los considerados en nuestro estudio como hiperglucemia (Os: glucemia $\geq 177 \mathrm{mg} / \mathrm{dl}$ ) lo que podría quizás explicar en nuestra muestra que la diferencia hallada en los pacientes hiperglucémicos no diabéticos fuera estadísticamente significativa y no así en los diabéticos conocidos.

En cuanto a la conducta adoptada en los pacientes para tratar la hiperglucemia, observamos que dos terceras partes de los hiperglucémicos (Qs = glucemia $>177 \mathrm{mg} / \mathrm{dl}$ ) recibieron tratamiento para su glucemia, quedando sin tratar una tercera parte de ellos. Con respecto a la conducta terapéutica de la glucemia según fueran diabéticos conocidos o no lo fueran, se detectó que 41 de 50 (82\%) diabéticos conocidos recibieron tratamiento para su glucemia, mientras que solamente a 10 de $27(41,8 \%)$ de los pacientes hiperglucémicos no conocidos como diabéticos les fue tratada la glucemia. De todos los pacientes tratados, casi el 90\% recibió insulina. Un 80\% de ellos recibió insulina por vía subcutánea y un 20\% por venoclisis. La media de glucemia de los tratados (tanto diabéticos como no conocidos) fue de $265,6 \pm 120,4 \mathrm{mg} / \mathrm{dl}$ (o sea un $50 \%$ más alta que el valor considerado en nuestro estudio como hiperglucemia [glucemia $>177 \mathrm{mg} / \mathrm{dll}$ ]), mientras que la media de los no tratados fue de $131,6 \pm 61,6 \mathrm{mg} / \mathrm{dl}$. Con respecto a las medias de glucemias según la vía de administración de la insulina para los tratados por vía subcutánea fue de $264 \pm 121,7 \mathrm{mg} / \mathrm{dl}$ y para la vía endovenosa 342,5 $\pm 94,8 \mathrm{mg} / \mathrm{dl}(p=0,002)$.

Estos datos expresan que, en ese momento (2000-2001), la tendencia de tratamiento de la glucemia fue en el $90 \%$ de los pacientes usar insulina (un $80 \%$ por vía subcutánea, casi seguramente por corrección por sliding scale como era la costumbre por aquellos años, y un $20 \%$ por vía intravenosa). Llamativamente hubo un 3,6\% con tratamiento de la glucemia por vía oral. La media de glucemia que se tuvo en cuenta para intervenir con insulina la glucemia fue un 50\% más alta que la que definimos como hiperglucemia en esta muestra y que la conducta médica no guió, fundamentalmente el valor de la glucemia, sino el hecho que los pacientes fueran diabéticos conocidos o no (se trató el $82 \%$ de los diabéticos vs el $41,8 \%$ de los no conocidos como diabéticos).

\section{CONCLUSIONES}

Aquí presentamos un estudio de corte transversal, retrospectivo, realizado en 11 hospitales generales del interior de Argentina, en el cual en 349 pacientes se analizó la mortalidad en IAM y su relación con una glucemia $\geq 177 \mathrm{mg} / \mathrm{dl}$ (Qs) considerada en nuestro análisis como hiperglucemia. Esta muestra nos mostró las mismas conclusiones que otros trabajos del mundo: que la hiperglucemia es un marcador de mortalidad en IAM y que esa diferencia es más evidente en pacientes que ingresan sin diagnóstico de diabetes que en diabéticos conocidos. Lo novedoso en nuestra muestra, ya que no ha sido observado por otros autores, es el hallazgo del aumento estadísticamente significativo de la tasa de mortalidad en pacientes hiperglucémicos con score 2 de la escala de Killip (insuficiencia cardíaca moderada).

En cuanto a la conducta terapéutica, observamos que una tercera parte de los hiperglucémicos no fue tratado, que los valores tenidos en cuenta para intervenir con insulina fueron un 50\% más altos que el valor definido como hiperglucemia en esta muestra y, por último, que para indicar tratamiento con insulina los médicos tomaron en cuenta fundamentalmente si los pacientes eran diabéticos conocidos o no, más que el valor de la glucemia hallada.

\section{Una puesta al día. Un poco de historia de lo que siguió nuestra experiencia}

A la fecha recolección de los datos de nuestro estudio aún no se habían publicado los trabajos que poco tiempo después definieron la necesidad de intervenir la glucemia en pacientes críticos para disminuir su morbimortalidad hospitalaria ${ }^{16,17,18}$.

La historia de ese cambio comenzó con la publicación del primer trabajo de Van den Berghe, et al. ${ }^{16}$, que obtuvo una reducción estadísticamente significativa de la mortalidad en UTI $(42,5 \%$ y $47,5 \%$ si permanecían en UTI más de cinco días) y hospitalaria global (34\%), y la mejora de varios parámetros clínicos y terapéuticos en el hospital. Ese trabajo de 2001 de Van den Berghe ${ }^{16}$ marcó un antes y después en el tema, pero hubo advertencias ya que el objetivo de normoglucemia $(80$ a $110 \mathrm{mg} / \mathrm{dl}$ ) planteaba dudas de su factibilidad en el mundo real. En 2004 Krinsley J, et al. ${ }^{17}$ hallaron que la intervención intensiva de la glucemia con objetivo $\leq 140 \mathrm{mg} / \mathrm{dl}$ comparado con un objetivo $\leq 200 \mathrm{mg} / \mathrm{dl}$ lograba una disminución de la mor- 
talidad hospitalaria de $29,3 \%(p=0,002)$ y una reducción de la estadía en UTI de 10,8\% ( $p=0,01)$, logros similares a los de Van den Berghe.

También se especuló si era posible extender esa experiencia a UTI generales (no quirúrgicas especialmente) y si el objetivo glucémico tan ajustado a la normoglucemia no produciría aumento de la tasa de hipoglucemias (aunque dicho estudio tenía solamente un 5,09\% de hipoglucemias [glucemia $<40 \mathrm{mg} / \mathrm{dll}$ ] en el grupo intensificado vs $0,76 \%$ en el convencional). La misma autora, hecha cuenta de la falta de pacientes de patologías no quirúrgicas en ese primer estudio (la crítica apuntaba a la mayor predictibilidad y preparación en los pacientes quirúrgicos de cardiocirugía que era el $62 \%$ de los pacientes enrolados), en 2006 publicó un estudio prospectivo y aleatorizado de pacientes en UTI general18. Allí los objetivos fueron idénticos pero no así los resultados. Las diferencias de mortalidad general no resultaron estadísticamente significativas entre ambos grupos ( $40 \%$ en el grupo de tratamiento convencional vs $37,3 \%$ en el intensificado) y lo que más llamó la atención fue que en los que permanecieron menos de tres días en UTI la mortalidad de los intensificados fue mayor, mientras que si la permanencia era de más de tres días era inversa: la mortalidad en los intensificados era significativamente menor que en los de tratamiento convencional $(52,5 \%$ vs $43 \%)$. Además la tasa de hipoglucemias en el grupo intensificado fue de $18,7 \%$ vs $3,1 \%$ en el convencional $(p<0,001)$, y la primera se elevaba a $25,1 \%$ en los de más de tres días de estadía en UTI $(p<0,001)$.

En años subsiguientes otros estudios expresaron resultados que aumentaron la preocupación acerca de si ese objetivo de normoglucemia estaba libre de riesgos para los pacientes. Basta con observar dos experiencias vividas por otros investigadores. La primera fue en 2008, cuando en el VISEP Trial ${ }^{19}$ la rama de tratamiento insulínico de la glucemia debió interrumpirse, pues en el grupo intensivo (80-110 mg/dl) hubo una tasa de hipoglucemias de $17,1 \%$ vs $4,1 \%$ y en el grupo convencional (140-180mg/dl), sin lograr con la intensificación de mejorías en la morbimortalidad en UTI. La segunda experiencia fue el Grucontrol Study ${ }^{20}$, trabajo prospectivo, aleatorizado, de UTI clínica-quirúrgica. El mismo debió interrumpirse con 1.101 pacientes enrolados por falta de beneficios clínicos de la rama intensificada (objetivo
80-110 mg/dl) y que se asoció a un aumento de hipoglucemia ( $8,7 \%$ vs $2,7 \%)$ cuando se comparó con el objetivo de 140-180 mg/dl.

Además por aquellos años estaba Ongoing, un trabajo prospectivo, aleatorizado, multicéntrico y multinacional: NICE-SUGAR Study, que se publicó en abril de 2009 en NEJM ${ }^{21}$. Este trabajo tuvo una rama intensiva (80-110 mg/dl) vs una convencional (140-180 mg/dl). El estudio enroló a 6.104 pacientes (3.054 en la rama intensiva y 3.050 en la convencional). La mortalidad global fue de $27,5 \%$ para el grupo intensivo vs $24,9 \%$ en el convencional $(O R=01,14$ [IC95\% 1,02-1,28] $p=0,02)$. No hubo diferencia en esas tasas de mortalidad si los pacientes eran quirúrgicos o clínicos $(\mathrm{ORq}=1,31$ vs $\mathrm{ORc}=1,07, \mathrm{p}=0,10)$. La tasa de hipoglucemias fue de $6,8 \%$ en el grupo intensivo vs $0,5 \%$ en el convencional $(p<0,001)$. No hubo diferencias significativas entre ambos grupos de tratamiento en cuanto a la media de días en UTI $(p=0,84)$, en el hospital $(0,86)$ o la media de días en respirador mecánico $(p=0,56)$ o días de hemodiálisis $(p=0,39)$. Si bien los mismos autores coincidían en que no podía aseverarse que un tratamiento intensivo de la glucemia fuera más agresivo que un tratamiento de objetivo de 140-180 mg/dl, alegaban que esa evidencia demostraba que el objetivo intensivo era menos seguro, pues no mejoraba los objetivos clínicos y aumentaba de forma significativa la tasa de hipoglucemias

Desde ese momento, basado en los hallazgos del NICE-SUGAR Study, las normativas de distintas sociedades científicas del mundo $22,23,24,25$ establecieron como objetivo para el paciente internado en UTI un valor de glucemia entre 140-180 mg/dl. En los Estándares de la Atención Médica en Diabetes 2018: Cuidado de la Diabetes en el Hospital ${ }^{26}$ de la ADA, se determinó:

- La hiperglucemia en el paciente internado se define como una glucemia $>140 \mathrm{mg} / \mathrm{dl}$.

- Debe iniciarse tratamiento insulínico por glucemias persistentes por encima del límite admitido de $\geq 180 \mathrm{mg} / \mathrm{dl}$.

- El objetivo de glucemia será mantenerlo entre 140 y $180 \mathrm{mg} / \mathrm{dl}$.

- Debemos considerar alerta de hipoglucemia con valores de glucemia $\leq 70 \mathrm{mg} / \mathrm{dl}$ e hipoglucemia clínicamente significativa con glucemia $\leq 54 \mathrm{mg} / \mathrm{dl}$.

En recientes estudios no se hallaron beneficios adicionales descendiendo los valores a menos de $140 \mathrm{mg} / \mathrm{dl}$, aún en pacientes quirúrgicos 27,28 , con- 
firmando los resultados obtenidos en 2009 por NICE-SUGAR ${ }^{21}$.

\section{Sobre la conducta terapéutica}

En cuanto a la conducta terapéutica, en la época de recolección de los datos de nuestra muestra, además de no tener objetivos glucémicos claros, basados en evidencias como las que posteriormente aparecieron, tampoco había evidencias que guiaran el tratamiento del paciente crítico con hiperglucemia.

En los trabajos de Van den Berghe ${ }^{16,18}$ y Krinsley ${ }^{17}$ se estableció que la terapéutica de la glucemia en el paciente crítico en UTI era con insulina por venoclisis, dejando de lado la insulinización por escala variable o más conocida como sliding scale, tan en uso en nuestra época de recolección de datos (en nuestro estudio observamos cómo el $80 \%$ de los insulinizados la recibía por vía subcutánea, ajustada seguramente por sliding scale). La insulinización por sliding scale está fuertemente desaconsejada, con nivel evidencia $A$, en los estándares de Atención Médica en Diabetes 2018 de la ADA, basándose en evidencias publicadas en diversos artículos $26,29,30$.

Existen diferentes protocolos publicados para implementar la secuenciación y seguimiento del tratamiento insulínico por venoclisis en UTI. Últimamente se publicó en Argentina un protocolo propuesto por médicos miembros de nuestra Sociedad $^{31}$, que es sencillo y accesible en la práctica de los médicos de UTI no especializados.

Luego de superada esa etapa se propuso una insulinización basada en esquema basal-bolo, de insulina basal más bolos de insulina rápida pre comidas, en una dosis total del 60 al $80 \%$ de la insulina recibida 24 hs previas por venoclisis ${ }^{31,32,33}$.

En el paciente no crítico internado en sala general se propuso, tanto en los Estándares de Atención Médica en Diabetes 2018 de ADA ${ }^{26}$ como en el artículo nacional citado ${ }^{31}$ y otros ${ }^{23,24,34}$, el uso de insulina en plan basal-bolo, estos pre comidas fijos o ajustados de acuerdo a escala variable o conteo de hidratos.

Actualmente la individualización de los objetivos y del tratamiento de los pacientes hiperglucémicos ha llegado hasta el paciente internado, sobre todo en el área no crítica. Para ello ya hay evidencias que esquemas de insulinización más "livianos" que el basal-bolo que pueden utilizarse en diferentes pacientes (obesos, añosos, riesgo
CV aumentado, entre otros) aún quirúrgicos ${ }^{35,36}$, como los esquemas basal plus, con corrección de insulina rápida en alguna comida. Últimamente se ha estudiado el uso de inhibidores de DPP-4 junto con la insulina basal para el manejo de la hiperglucemia en estos pacientes ${ }^{37,38}$. También existe cierta evidencia con análogos de GLP-1 que recién comienza a aparecer ${ }^{39,40}$. Aún estos usos no han sido aprobados por las agencias de medicamentos como la Food and Drug Administration (FDA), por ejemplo ${ }^{41}$.

\section{CONCLUSIONES FINALES}

Nuestro trabajo aportó datos interesantes acerca de la mortalidad por infarto y su relación con la presencia de hiperglucemia al ingreso hospitalario, tanto en pacientes diabéticos conocidos como si no lo eran. Además, sumó datos acerca de la conducta terapéutica de los médicos ante la hiperglucemia en aquel momento. Tanta agua ha pasado bajo este puente en estos años que ha transformado estos resultados en algo así como un aporte meramente histórico. Por ello es que planteamos desde esta publicación, la movilización de investigadores de nuestra Sociedad para evaluar en un nuevo trabajo cuánto han cambiado, en el mundo real, los resultados y las conductas ante este tipo de pacientes.

\section{BIBLIOGRAFÍA}

1. Cruikshank N. Coronary thrombosis and myocardial infarction with glycosuria. BMJ 1931; 1: 618-19.

2. Yudkin JS, Oswald GA. Hyperglycaemia, diabetes and myocardial infarction. Diabetes Med 1987; 4: 13-18.

3. Malmberg K, Ryden L, Efendic S, et al. Randomized trial of insulin-glucose infusion followed by subcutaneous insulin treatment in diabetic patients with acute myocardial infarction (DIGAMI study). Effects on mortality at 1 year. J Am Coll Cardiol 1995; 26: 57-65.

4. Capes SE, Hunt D, Malmberg K, Gerstein HC. Stress hyperglycemia and increased risk of death after myocardial infarction in patients with and without diabetes: a systematic overview. Lancet 2000; 355: 773-778.

5. Inzucchi SE, Rosenstock J. Counterpoint: inpatient glucose management. Diabetes Care 2005; 28: 976-979.

6. McMahon MM, Bistrian BR. Host defenses and susceptibility to infection in patients with diabetes mellitus. Infect Dis Clin North Am 1995; 9: 1-9.

7. Delamaire M, Maugendre D, Moreno M, Le Goff MC, Allannic H, Genetet CG. Impaired leucocytes functions in diabetic patients. Diabet Med 1997; 14: 29-34.

8. Kersten J, Schmeling T, Orth K, Warltier D. Acute hyperglycemia abolishes ischemic preconditioning in vivo. Am J Physiol 1998; 275: H721-H725. 
9. Ceriello A, Quagliaro L, D'Amico M, Di Filippo C, Marfella R, Nappo F, Berrino L, Rossi F, Giugliano D. Acute hyperglycemia induces nitrotyrosine formation and apoptosis in perfused heart from rat. Diabetes 2002; 51: 1076-1082.

10. Pulsinelli WA, Waldman S, Rawlinson D, Plum F. Moderate hyperglycemia augments ischemic brain damage: a neuropathologic study in the rat. Neurology 1982; 32: 1239-1246.

11. Dieuzeide G, Viñes G, Salazar-Sáez E, Arrieta J, Sáenz R, Mayas A, Sosa A, Santillán C, Bauchi JC, Rimoli D, Alzueta G, Bellusci N, Denaro R, Luquesi M, Vera O, Salazar R, González C. Valor pronóstico de la hiperglucemia al ingreso hospitalario en la mortalidad por accidente cerebrovascular. Rev Soc Arg de Diabetes 2005; 39: 3-9.

12. Williams S, Goldfine A, Timimi F, Ting H, Roddy M, Simonson D, Creager M. Acute hyperglycemia attenuates endothelium-dependent vasodilatation in humans in vivo. Circulation 1998; 97: 1695-1701.

13. Oliver MF, Opie LH. Effects of glucose and fatty acids on myocardial ischaemia and arrhythmias. Lancet 1994; 343: 155-158.

14. Mjos OD. Effect of free fatty acids on myocardial function and oxygen consumption in intact dogs. J Clin Invest 1971; 50: 1386-89.

15. Malmberg K, Norhammar A, Wedel H, Ryden L. Glycometabolic state at admission: important risk marker of mortality in conventionally treated patients with diabetes mellitus and acute myocardial infarction. Circulation 1999; 99: 2626-2632.

16. Van den Berghe G, Wouters P, Weekers F, Verwaest C, Bruyninckx F, Schetz M, Vlasselaers D, Ferdinande $P$, Lauwers P, Bouillon R. Intensive insulin therapy in critically III patients. N Engl J Med 2001; 345:1359-1367.

17. Krinsley JS. Effect of an intensive glucose management protocol on the mortality of critically ill adult patients. Mayo Clinic Proceedings August 2004; Vol 79 (8), 992-1000.

18. Van den Berghe G, Wilmer A, Hermans G, Meersseman W, Wouters PJ, Milants I, Van Wijngaerden E, Bobbaers $\mathrm{H}$, Bouillon R. Intensive insulin therapy in the medical ICU. N Engl J Med 2006; 354:449-461.

19. Brunkhorst FM, Engel C, Bloos F, Meier-Hellmann A, Ragaller M, Weiler N, Moerer O, Gruendling M, Oppert M, Grond S, Olthoff D, Jaschinski U, Rossaint JS, Welte R, SchaeferT, Kern M. Study protocol of the VISEP study. Response of the SepNet study group. Intensive insulin therapy and pentastarch. Resuscitation in severe sepsis. N Engl J Med 2008; 358:125-39.

20. Preiser JC, Devos P, Ruiz-Santana S, Mélot C, Annane D, Groeneveld J, lapichino G, Leverve X, Nitenberg G, Singer P, Wernerman J, Joannidis M, Stecher A, Chioléro R. A prospective randomised multi-centre controlled trial on tight glucose control by intensive insulin therapy in adult intensive care units: the Glucontrol Study. Intensive Care Med 2009 Oct; 35(10):1738-48.

21. The NICE-SUGAR Study Investigators. Intensive vs conventional glucose control in critically III patients. N Engl J Med 2009; 360:1283-1297.

22. Moghissi ES, Korytkowski MT, DiNardo M, et al.; American Association of Clinical Endocrinologists, American Diabetes Association. Consensus statement on inpatient glycemic control. Diabetes Care 2009;32:1119-1131.
23. Qaseem A, Humphrey LL, Chou R, Snow V, Shekelle $\mathrm{P}$; Clinical Guidelines Committee of the American College of Physicians. Use of intensive insulin therapy for the management of glycemic control in hospitalized patients: a clinical practice guideline from the American College of Physicians. Ann Intern Med 2011; 154:260-267

24. Umpierrez GE, Hellman R, Korytkowski MT, et al.; Endocrine Society. Management of hyperglycemia in hospitalized patients in non-critical care setting: an Endocrine Society clinical practice guideline. J Clin Endocrinol Metab 2012; 97:16-38.

25. Jacobi J, Bircher N, Krinsley J, et al. Guidelines for the use of an insulin infusion for the management of hyperglycemia in critically ill patients. Crit Care Med 2012; 40:3251-3276.

26. Diabetes Care in the Hospital: Standards of Medical Care in Diabetes 2018 American Diabetes Association. Diabetes Care 2018 Jan; 41(Supp 1): S144-S151.

27. Sathya B, Davis R, Taveira T, Whitlatch H, Wu W-C. Intensity of peri-operative glycemic control and postoperative outcomes in patients with diabetes: a metaanalysis. Diabetes Res Clin Pract 2013; 102:8-15.

28. Umpierrez G, Cardona S, Pasquel F, et al. Randomized controlled trial of intensive versus conservative glucose control in patients undergoing coronary artery bypass graft surgery: GLUCO-CABG trial. Diabetes Care 2015; 38:1665-1672.

29. Moghissi ES, Korytkowski MT, DiNardo M, et al.; American Association of Clinical Endocrinologists, American Diabetes Association. Consensus statement on inpatient glycemic control. Diabetes Care 2009; 32:1119-1131.

30. Draznin B, Gilden J, Golden SH, et al.; PRIDE investigators. Pathways to quality inpatient management of hyperglycemia and diabetes: a call to action. Diabetes Care 2013; 36:1807-1814.

31. Grosembacher LA, Puchulu F, Fretes O, Giunta J, González C, Umpierrez G. Guía de recomendaciones para el manejo de la hiperglucemia en pacientes hospitalizados. Federación Argentina de Sociedades de Endocrinología (FASEN) 2016. Rev Endocrinol Metab 2018; 55(1): 34-40.

32. Schmeltz LR, DeSantis AJ, Thiyagarajan V, et al. Reduction of surgical mortality and morbidity in diabetic patients undergoing cardiac surgery with a combined intravenous and subcutaneous insulin glucose management strategy. Diabetes Care 2007; 30:823-828.

33. Shomali ME, Herr DL, Hill PC, Pehlivanova M, Sharretts JM, Magee MF. Conversion from intravenous insulin to subcutaneous insulin after cardiovascular surgery: transition to target study. Diabetes Technol Ther 2011;13:121-126.

34. Umpierrez GE, Smiley D, Jacobs S, et al. Randomized study of basal-bolus insulin therapy in the inpatient management of patients with type 2 diabetes undergoing general surgery (RABBIT 2 surgery). Diabetes Care 2011; 34:256-261.

35. Umpierrez GE, et al. Randomized study comparing a basal-bolus with a basal plus correction insulin regimen for the hospital management of medical and surgical patients with type 2 diabetes: basal plus trial. Diabetes Care 2013 Aug; 36(8):2169-74. 
36. Smiley $D$, et al. Differences in inpatient glycemic control and response to subcutaneous insulin therapy between medicine and surgery patients with type 2 diabetes. J Diabetes Complications 2013 Nov-Dec; 27(6):637-41.

37. Umpierrez GE, Gianchandani R, Smiley D, et al. Safety and efficacy of sitagliptin therapy for the inpatient management of general medicine and surgery patients with type 2 diabetes: a pilot, randomized, controlled study. Diabetes Care 2013; 36:3430-3435.

38. Pasquel FJ, et al. Efficacy of sitagliptin for the hospital management of general medicine and surgery patients with type 2 diabetes (Sita-Hospital): a multicentre, prospective, open-label, non-inferiority randomised trial. Lancet Diabetes \& Endocrinology 2017; 5 (2) $125-133$.
39. Mendez CE, Umpierrez GE. Pharmacotherapy for hyperglycemia in noncritically ill hospitalized patients. Diabetes Spectr 2014; 27:180-188.

40. Umpierrez GE, Korytkowski M. Is incretin-based therapy ready for the care of hospitalized patients with type 2 diabetes? Insulin therapy has proven itself and is considered the mainstay of treatment. Diabetes Care 2013; 36:2112-2117.

41. U.S. Food and Drug Administration. FDA Drug Safety Communication: FDA adds warnings about heart failure risk to labels of type 2 diabetes medicines containing saxagliptin and alogliptin (Internet), 2016. Disponible en: http://www.fda.gov/Drugs/DrugSafety/ucm486096. htm. Acceso: 7 de octubre de 2016. 